\title{
Aproximación a valores de referencia de estudios electrofisiológicos para el diagnóstico de Síndrome de Túnel del Carpo
} Using neurophysiological reference values as an approach to carpal tunnel syndrome diagnosis

Fernando Ortiz-Corredor ${ }^{1,2,3}$ y Ángela López-Monsalve $e^{1,4}$

\author{
1 Departamento de Medicina Física y Rehabilitación, Facultad de Medicina, Universidad Nacional de \\ Colombia. Bogotá. fortizc@unal.edu.co \\ 2 Centro de Investigación en Fisiatría y Electrodiagnóstico CIFEL. \\ 3 Instituto de Ortopedia Infantil Roosevelt. Bogotá, Colombia \\ 4 Hospital Central de la Policía. Bogotá, Colombia
}

Recibido 26 Febrero 2009/Enviado para Modificación 8 Agosto 2009/Aceptado 5 Septiembre

\section{RESUMEN}

Objetivo Establecer valores normales de referencia de latencias distales motoras y sensitivas de los nervios mediano y ulnar para el diagnóstico electrofisiológico de síndrome de túnel del carpo (STC) teniendo en cuenta las características demográficas de nuestro medio.

Métodos Se realizaron estudios de neuroconducción sensitiva y motora de los nervios mediano y ulnar en 184 individuos asintomáticos (rango de edad 18 a 75 años). Con los resultados se construyeron tablas de referencia con promedios, desviaciones estándar y percentiles y se compararon con edad, género, talla y peso.

Resultados Para el nervio mediano se encontró una latencia motora distal y sensitiva al pico promedio de $3.4 \mathrm{~ms}(\mathrm{DE}=0.4)$ y $3.1 \mathrm{~ms}(\mathrm{DE}=0.3)$, respectivamente (prueba convencional). La diferencia de la latencia motora mediano-ulnar fue de $0.8 \mathrm{~ms}$ $(\mathrm{DE}=0.3)$; la diferencia de la latencia sensitiva mediano-ulnar al pico fue de $0.08 \mathrm{~ms}$ $(\mathrm{DE}=0.2)$ (prueba convencional). La latencia motora y sensitiva del nervio mediano mostró una correlación positiva con la edad y la talla.

Conclusiones Aunque los resultados de este estudio tienen un alcance limitado y preliminar por el tipo y tamaño de muestra utilizado, éstos muestran algunas diferencias con los datos obtenidos en investigaciones de otros países y sirven como una primera guía para el diagnóstico de STC en un laboratorio de electrofisiología en nuestro medio.

Palabras Clave: Electrodiagnóstico, valores de referencia, Síndrome del Túnel Carpiano (fuente: DeCS, BIREME).

\section{ABSTRACT}

Objective Establishing normal reference values for median and ulnar nerve distal motor and sensory latencies for electrophysiological diagnosis of carpal tunnel syndrome (CTS) taking our population's demographic traits into account. 
Methods Sensory and motor nerve conduction studies for the median and ulnar nerves were performed on 184 non-symptomatic people (age range: 18-75). Reference tables (including means, standard deviations and percentiles) were constructed from the findings. The results were compared to demographic variables such as age, gender, height and weight.

Results 3.4 ms mean distal motor latency $(S D=0.4)$ and $3.1 \mathrm{~ms}$ sensory peak latency $(S D=0.3)$ were found for the median nerve (conventional test). $0.8 \mathrm{~ms}$ distal motor median-ulnar latency difference $(S D=0.2)$ and $0.08 \mathrm{~ms}$ mean sensory median-ulnar peak latency difference $(S D=0.2)$ were found (conventional test). Motor and sensory median nerve latency showed a positive relationship with age and height.

Conclusions Even though the present study's results have a restricted and preliminary impact due to the type and size of the sample used, they did reveal some differences from data obtained in other countries and could be useful as an initial guide for CTS diagnosis in a Colombian electrophysiological laboratory.

Key Words: Electrodiagnosis, reference value, carpal tunnel syndrome (source: $\mathrm{MeSH}$, NLM).

$\mathrm{E}$

1 Síndrome de Túnel del Carpo (STC) es un diagnóstico frecuente en medicina ocupacional y en el nivel básico de atención en salud. Los criterios de anormalidad electrofisiológica para el diagnóstico de STC se basan en la comparación de los resultados del examen con los valores normales de las latencias sensitivas y motoras del nervio mediano y cubital de personas sanas. Para el diagnóstico de diferentes patologías neuromusculares se deben utilizar valores de referencia de individuos con características antropométricas similares a las que presentan los pacientes. En nuestro medio, no existen valores de referencia para el diagnóstico electrofisiológico del STC y en los laboratorios se utilizan como guía las tablas publicadas en otros países.

El objetivo del presente estudio es construir tablas preliminares de referencia para valores obtenidos en exámenes de neuroconducción de los nervios mediano y ulnar en adultos asintomáticos de diferentes grupos de edad.

\section{MÉTODOS}

El estudio se realizó en la Clínica Carlos Lleras Restrepo, Bogotá-Colombia. Los valores de referencia fueron obtenidos en 184 individuos asintomáticos (estudiantes de medicina, médicos, personal de enfermería, acompañantes de los pacientes) sin antecedentes de diabetes, hipotiroidismo, alcoholismo, cervicobraquialgia y osteoartritis. Adicionalmente se obtuvo el peso, la talla. 
Se compararon los valores obtenidos para cada parámetro electrofisiológico entre los pacientes mayores y menores de 40 años. La división se basó en que el diagnóstico clínico de STC es muy común después de los 40 años de edad y raro en pacientes más jóvenes.

Electrofisiología. En todos los individuos, se examinó el miembro superior derecho y la temperatura en la extremidad se controló para que no estuviera por debajo de $32^{\circ} \mathrm{C}$.

Para las neuroconducciones motoras del nervio mediano y ulnar, el electrodo activo se colocó en el abductor pollicis brevis y en el abductor digiti minimi, respectivamente, estimulando a $8 \mathrm{~cm}$ en el trayecto de los nervios.

Para las neuroconducciones sensitivas convencionales se realizó la prueba registrando en los dedos $2^{\circ}$ y $5^{\circ}$ estimulando a $14 \mathrm{~cm}$ los nervios mediano y ulnar, respectivamente. Para la prueba comparativa se registró en el $4^{\circ}$ dedo y se estimuló a $14 \mathrm{~cm}$ en el trayecto de los nervios.

Análisis estadístico. Se calcularon promedios con desviaciones estándar y percentiles para cada uno de los parámetros electrofisiológicos. Se realizó un análisis bivariado de correlación lineal entre la edad, peso, talla y los diferentes resultados electrofisiológicos utilizando la prueba de Spearman. Adicionalmente se llevó a cabo un análisis de regresión lineal múltiple para determinar las variables independientes asociadas con los resultados. En este caso se realizaron transformaciones de los datos para obtener una distribución normal (1).

\section{RESULTADOS}

Se evaluaron 184 individuos, 111 de 40 o más años, $51.9 \%$ de sexo femenino (Tabla 1). El promedio de edad para los hombres fue de 44.2 años (DE=16.4) y 42.3 años para las mujeres ( $\mathrm{DE}=14.1)$. La estatura promedio para los hombres fue $1.69 \mathrm{~cm}(\mathrm{DE}=0.06)$ y $1.57 \mathrm{~cm}$ para las mujeres $(\mathrm{DE}=0.05)$. Los resultados se muestran en las Tablas 1,2 y 3 .

La latencia motora del mediano fue más alta en hombres (promedio en mujeres 3,2 ms; promedio en hombres $3,5 \mathrm{~ms} ; \mathrm{p}=0,000$ ). Así mismo, los valores aumentaron en relación directa con la edad $(\mathrm{r}=0,3 ; \mathrm{p}=0,000)$, el peso $(\mathrm{r}=0,3$; $\mathrm{p}=0,000)$ y la talla $(\mathrm{r}=0,2 ; \mathrm{p}=0,007)$. En el análisis de regresión lineal múltiple se encontró una correlación significativa con la edad y la talla $(\mathrm{p}=0,000)$. 
Para la diferencia motora mediano-ulnar la edad mostró una correlación significativa $(r=0,2 ; \mathrm{p}=0,002)$.

Tabla 1. Latencias sensitivas y motoras del nervio mediano y ulnar en individuos de todos los grupos de edad

\begin{tabular}{|c|c|c|c|c|c|c|}
\hline & Mínimo & Máximo & Promedio & $\begin{array}{c}\text { Desviación } \\
\text { Estándar }\end{array}$ & $\begin{array}{c}\text { Promedio + } \\
\text { 2DE }\end{array}$ & $\begin{array}{c}\text { Percentil } \\
97\end{array}$ \\
\hline $\begin{array}{l}\text { Latencia mediano } \\
\text { motor }\end{array}$ & 2,5 & 5,9 & 3,4 & 0,4 & 4,2 & 4,3 \\
\hline $\begin{array}{l}\text { Latencia ulnar } \\
\text { motor }\end{array}$ & 2,2 & 3,7 & 2,6 & 0,2 & 3,1 & 3,2 \\
\hline $\begin{array}{l}\text { Diferencia latencia } \\
\text { motora mediano- } \\
\text { ulnar }\end{array}$ & $-0,1$ & 2,0 & 0,8 & 0,3 & 1,5 & 1,4 \\
\hline $\begin{array}{l}\text { Latencia mediano } \\
\text { sensitiva al inicio }\end{array}$ & 1,8 & 3,5 & 2,4 & 0,3 & 3,0 & 3,1 \\
\hline $\begin{array}{l}\text { Latencia mediano } \\
\text { sensitiva al pico }\end{array}$ & 2,5 & 4,2 & 3,1 & 0,3 & 3,7 & 4,0 \\
\hline $\begin{array}{l}\text { Latencia Ulnar } \\
\text { sensitivo al inicio }\end{array}$ & 2,0 & 3,1 & 2,4 & 0,2 & 2,9 & 2,9 \\
\hline $\begin{array}{l}\text { Latencia Ulnar } \\
\text { sensitivo al pico }\end{array}$ & 2,4 & 3,9 & 3,0 & 0,2 & 3,6 & 3,6 \\
\hline $\begin{array}{l}\text { Diferencia sensitiva } \\
\text { mediano ulnar } \\
\text { convencional inicio }\end{array}$ & $-0,6$ & 1,3 & 0,0 & 0,2 & 0,7 & 0,7 \\
\hline $\begin{array}{l}\text { Diferencia sensitiva } \\
\text { mediano ulnar } \\
\text { convencional pico }\end{array}$ & $-0,4$ & 1,0 & 0,0 & 0,2 & 0,6 & 0,7 \\
\hline $\begin{array}{l}\text { Diferencia sensitiva } \\
\text { mediano ulnar } \\
\text { cuarto dedo inicio }\end{array}$ & $-0,5$ & 1,1 & 0,1 & 0,2 & 0,7 & 0,8 \\
\hline $\begin{array}{l}\text { Diferencia sensitiva } \\
\text { mediano ulnar } \\
\text { cuarto dedo pico }\end{array}$ & $-0,9$ & 1,3 & 0,1 & 0,2 & 0,7 & 0,7 \\
\hline
\end{tabular}

Las latencias sensitivas del nervio mediano al inicio y al pico del potencial mostraron una distribución asimétrica positiva. Así, para el análisis multivariado se realizó una transformación $1 /(\mathrm{X})$, donde $\mathrm{X}=$ latencia sensitiva del nervio mediano al inicio o pico del potencial. Los resultados transformados del inicio del potencial se encontraron inversamente correlacionados con la edad y el peso. En el análisis multivariado se encontró una correlación significativa con la edad $(\mathrm{R} 2=0,1 ; \mathrm{p}=0,000)$. Los resultados transformados del pico del potencial mostraron una correlación inversa con el peso $(\mathrm{r}=-0,2)$ y la edad $(\mathrm{r}=-0,3)$. El análisis multivariado mostró una correlación significativa de la latencia sensitiva con la edad y el peso con una R2 baja $(0,1)$.

Entre los individuos mayores y menores de 40 años se encontró una diferencia significativa para las latencias motoras $(\mathrm{p}=0,000)$ y sensitivas $(\mathrm{p}=0,000)$ del nervio mediano y para la prueba comparativa mediano-ulnar en el $4^{\circ}$ dedo $(\mathrm{p}=0,002)$. 
Tabla 2. Latencias sensitivas y motoras del nervio mediano y ulnar en individuos menores de 40 años

\begin{tabular}{|c|c|c|c|c|c|c|}
\hline & Mínimo & Máximo & Promedio & $\begin{array}{c}\text { Desviación } \\
\text { Estándar }\end{array}$ & $\begin{array}{c}\text { Promedio + } \\
2 \mathrm{DE}\end{array}$ & $\begin{array}{c}\text { Percentil } \\
97\end{array}$ \\
\hline $\begin{array}{l}\text { Latencia mediano } \\
\text { motor }\end{array}$ & 2,5 & 4,2 & 3,2 & 0,3 & 3,9 & 4,0 \\
\hline $\begin{array}{l}\text { Latencia ulnar } \\
\text { motor }\end{array}$ & 2,2 & 3,3 & 2,6 & 0,2 & 3,1 & 3,2 \\
\hline $\begin{array}{l}\text { Diferencia latencia } \\
\text { motora mediano- } \\
\text { ulnar }\end{array}$ & $-0,1$ & 1,4 &, 59 & 0,3 & 1,1 & 1,3 \\
\hline $\begin{array}{l}\text { Latencia mediano } \\
\text { sensitiva al inicio }\end{array}$ & 1,8 & 3,4 & 2,3 & 0,2 & 2,9 & 3,1 \\
\hline $\begin{array}{l}\text { Latencia mediano } \\
\text { sensitiva al pico }\end{array}$ & 2,5 & 4,2 & 3,0 & 0,3 & 3,6 & 3,9 \\
\hline $\begin{array}{l}\text { Latencia Ulnar } \\
\text { sensitivo al inicio }\end{array}$ & 2,0 & 3,0 & 2,3 & 0,2 & 2,8 & 2,9 \\
\hline $\begin{array}{l}\text { Latencia Ulnar } \\
\text { sensitivo al pico }\end{array}$ & 2,5 & 3,7 & 3,0 & 0,2 & 3,5 & 3,6 \\
\hline $\begin{array}{l}\text { Diferencia sensitiva } \\
\text { mediano ulnar } \\
\text { convencional inicio }\end{array}$ & $-0,5$ & 0,8 & 0,0 & 0,2 & 0,5 & 0,6 \\
\hline $\begin{array}{l}\text { Diferencia sensitiva } \\
\text { mediano ulnar } \\
\text { convencional pico }\end{array}$ & $-0,4$ & 0,7 & 0,0 & 0,2 & 0,5 & 0,7 \\
\hline $\begin{array}{l}\text { Diferencia sensitiva } \\
\text { mediano ulnar } \\
\text { cuarto dedo inicio }\end{array}$ & $-0,5$ & 1,1 & 0,0 & 0,2 & 0,6 & 0,9 \\
\hline $\begin{array}{l}\text { Diferencia sensitiva } \\
\text { mediano ulnar } \\
\text { cuarto dedo pico } \\
\end{array}$ & $-0,9$ & 1,3 & 0,0 & 0,2 & 0,6 & 0,8 \\
\hline
\end{tabular}

\section{DISCUSIÓN}

Los valores obtenidos en nuestro estudio no son representativos de ninguna población, pero sirven como una primera aproximación para Colombia de los datos que se pueden obtener en individuos asintomáticos. Además de los factores técnicos ampliamente conocidos, los resultados de estudios de neuroconducción practicados a individuos asintomáticos dependen de factores antropométricos y probablemente socioeconómicos. Por ejemplo, aunque las edades en nuestro estudio son similares a las referidas en la investigación de valores normales de Buschbacher, la talla y el peso son significativamente diferentes $(\mathrm{p}=0,000)(2)$.

Los límites de normalidad en nuestros controles coinciden con los reportados por Melvin, Chang y Atroshi (3-5). El hallazgo más importante es que las latencias motoras y sensitivas del nervio mediano aumentan con la edad lo cual sugiere que en algunas personas se puede producir una neuropatía asintomática en el túnel del carpo. Existen diferencias significativas en los tiempos de latencia distal del nervio mediano y ulnar entre las personas mayores y menores de 40 años. Nuestro estudio mostró que las latencias motoras se prolongaron en una 
relación directa con la edad encontrando frecuentemente valores para el nervio mediano por encima de 4,0 ms en personas mayores de 50 años. La latencia motora del nervio mediano para los adultos mayores de 60 años fue de $3,5 \mathrm{~ms}$ $(\mathrm{DE}=0,3)$ que comparado con la latencia de $3,3 \mathrm{~ms}$ en individuos de menor edad deja una significancia estadística marginal de 0,05 . Por el contrario, la comparación de la latencia motora del nervio ulnar entre los diferentes grupos de edad no mostró diferencias significativas, Otros autores no apoyan la hipótesis de los atrapamientos asintomáticos. Falco estudió 155 adultos mayores de 60 años de edad y solo encontró una correlación directa de la edad con las latencias y velocidades del nervio ulnar (6). Este autor propuso que las latencias motoras prolongadas observadas en individuos mayores de 60 años, se debía a que en este grupo de edad el control de la temperatura en las extremidades era más difícil. Sin embargo, esta teoría no explica la razón por la cual la latencia motora del nervio ulnar no se afecta en la misma medida que el nervio mediano.

Los valores de las neuroconducciones sensitivas requieren especial atención ya que son las primeras en mostrar anormalidades en los pacientes con STC. La latencia sensitiva promedio y los rangos de normalidad para la técnica convencional se aproximan a los encontrados por otros autores $(4,5,7,8)$. En el presente estudio, las latencias sensitivas mostraron una distribución asimétrica positiva. Una posibilidad, similar a la planteada para la latencia motora del nervio mediano, es que en algunas personas las latencias prolongadas sean causadas por una neuropatía asintomática que produce valores extremos positivos (9). Como en el caso de las latencias motoras, en nuestro grupo de 27 individuos mayores de 60 años, las latencias sensitivas se encontraron significativamente mayores que en personas menores.

La evaluación del nervio mediano en el $4^{\circ}$ dedo con una diferencia recomendada de 0,4 ms del nervio mediano con el nervio ulnar, ha sido considerada una de las pruebas más sensibles para el diagnóstico del STC $(7,10)$. Ese valor es utilizado por la mayoría de electromiografistas de nuestro país. Nuestros datos de normalidad con el límite en $0,7 \mathrm{~ms}$ coinciden con los valores de otros autores $(3,8)$. En la práctica cotidiana, un examen que muestra una diferencia en las latencias sensitivas del nervio mediano con el nervio ulnar de 0,5 milisegundos es informado como anormal en la mayoría de laboratorios del país pero de acuerdo a nuestros valores de referencia, este mismo valor sería interpretado como normal. A diferencia del valor de corte de 0,4 milisegundos, un valor de corte de 0,7 milisegundos disminuye la sensibilidad de la prueba pero la hace más específica. 
Tabla 3. Latencias sensitivas y motoras del nervio mediano y ulnar en individuos mayores de 40 años

\begin{tabular}{|c|c|c|c|c|c|c|}
\hline & Mínimo & Máximo & Promedio & $\begin{array}{l}\text { Desviación } \\
\text { Estándar }\end{array}$ & $\begin{array}{l}\text { Promedio + } \\
\text { 2DE }\end{array}$ & $\begin{array}{l}\text { Percentil } \\
\quad 97\end{array}$ \\
\hline $\begin{array}{l}\text { Latencia mediano } \\
\text { motor }\end{array}$ & 2,7 & 4,6 & 3,5 & 0,4 & 4,3 & 4,4 \\
\hline $\begin{array}{l}\text { Latencia ulnar } \\
\text { motor }\end{array}$ & 2,2 & 3,7 & 2,7 & 0,3 & 3,2 & 3,2 \\
\hline $\begin{array}{l}\text { Diferencia latencia } \\
\text { motora mediano- } \\
\text { ulnar }\end{array}$ & , 10 & 2,0 & 0,8 & 0,3 & 1,5 & 1,5 \\
\hline $\begin{array}{l}\text { Latencia mediano } \\
\text { sensitiva al inicio }\end{array}$ & 1,9 & 3,5 & 2,5 & 0,3 & 3,0 & 3,2 \\
\hline $\begin{array}{l}\text { Latencia mediano } \\
\text { sensitiva al pico }\end{array}$ & 2,5 & 4,2 & 3,2 & 0,3 & 3,9 & 4,0 \\
\hline $\begin{array}{l}\text { Latencia Ulnar } \\
\text { sensitivo al inicio }\end{array}$ & 2,0 & 3,1 & 2,4 & 0,2 & 2,9 & 2,9 \\
\hline $\begin{array}{l}\text { Latencia Ulnar } \\
\text { sensitivo al pico }\end{array}$ & 2,4 & 3,9 & 3,0 & 0,3 & 3,6 & 3,8 \\
\hline $\begin{array}{l}\text { Diferencia sensitiva } \\
\text { mediano ulnar } \\
\text { convencional inicio }\end{array}$ & $-0,6$ & 1,3 & 0,1 & 0,3 & 0,7 & 0,8 \\
\hline $\begin{array}{l}\text { Diferencia sensitiva } \\
\text { mediano ulnar } \\
\text { convencional pico }\end{array}$ & $-0,4$ & 1,0 & 0,1 & 0,2 & 0,7 & 0,8 \\
\hline $\begin{array}{l}\text { Diferencia sensitiva } \\
\text { mediano ulnar } \\
\text { cuarto dedo inicio }\end{array}$ & $-0,3$ & 0,8 & 0,2 & 0,2 & 0,7 & 0,8 \\
\hline $\begin{array}{l}\text { Diferencia sensitiva } \\
\text { mediano ulnar } \\
\text { cuarto dedo pico }\end{array}$ & $-0,5$ & 0,8 & 0,1 & 0,2 & 0,7 & 0,7 \\
\hline
\end{tabular}

En los laboratorios de electrofisiología de nuestro país seemplean otras técnicas tales como el estudio ortodrómico sensitivo mixto del nervio mediano y ulnar con estimulación mediopalmar o la prueba comparativa del nervio mediano con el nervio radial en el primer dedo. No se han publicado en nuestro país valores de referencia de estas técnicas.

En resumen, aunque los datos obtenidos no son representativos de la población, este estudio muestra una primera aproximación a valores de referencia que se pueden obtener en un laboratorio de electrofisiología de nuestro medio para el estudio de pacientes con posible STC, con las características antropométricas de nuestra población. Hacia el futuro, las investigaciones se deben dirigir a obtener valores de referencia a partir de muestras de grupos poblacionales específicos más grandes de acuerdo a la edad y la ocupación, y a establecer los niveles de sensibilidad, especificidad y valores predicativos para las diferentes pruebas que se utilizan frecuentemente en los laboratorios de electrofisiología con el fin de buscar abordajes diagnósticos estandarizados que permitan establecer indicaciones terapéuticas homogéneas y comparables 
Agradecimientos. A Camilo Mendoza-Pulido por su asistencia editorial.

\section{REFERENCIAS}

1. Campbell WW, Robinson LR. Deriving reference values in electrodiagnostic medicine. Muscle Nerve. 1993;16:424-8.

2. Buschbacher RM. Median nerve motor conduction to the abductor pollicis brevis. Am J Phys Med Rehabil. 1999;78(6 Suppl):S1-S8.

3. Atroshi I, Gummesson C, Johnsson R, Ornstein E. Diagnostic properties of nerve conduction tests in population-based carpal tunnel syndrome. BMC Musculoskelet Disord. 2003;4:9.

4. Chang MH, Wei SJ, Chiang HL, Wang HM, Hsieh PF, Huang SY. Comparison of motor conduction techniques in the diagnosis of carpal tunnel syndrome. Neurology. 2002; 58:1603-7.

5. Melvin JL, Schuchmann JA, Lanese RR. Diagnostic specificity of motor and sensory nerve conduction variables in the carpal tunnel syndrome. Arch Phys Med Rehabil. 1973; 54:69-74.

6. Falco FJ, Hennessey WJ, Braddom RL, Goldberg G. Standardized nerve conduction studies in the upper limb of the healthy elderly. Am J Phys Med Rehabil. 1992;71:263-71.

7. Jackson DA, Clifford JC. Electrodiagnosis of mild carpal tunnel syndrome. Arch Phys Med Rehabil. 1989;70:199-204.

8. Salerno DF, Franzblau A, Werner RA, Bromberg MB, Armstrong TJ, Albers JW. Median and ulnar nerve conduction studies among workers: normative values. Muscle Nerve.1998; 21:9991005.

9. Robinson LR, Temkin NR, Fujimoto WY, Stolov WC. Effect of statistical methodology on normal limits in nerve conduction studies. Muscle Nerve. 1991;14:1084-90.

10. Johnson EW, Kukla RD, Wongsam PE, Piedmont A. Sensory latencies to the ring finger: normal values and relation to carpal tunnel syndrome. Arch Phys Med Rehabil. 1981;62:206-8. 